\title{
Seleção de métodos para modularização no desenvolvimento de produtos: revisão sistemática
}

\author{
Monique Sonego $0^{a^{*}}$, Márcia Soares Echeveste ${ }^{a^{* *}}$ \\ aniversidade Federal do Rio Grande do Sul, Porto Alegre, RS, Brasil \\ *hgmonique@gmail.com, ***echeveste@producao.ufrgs.br
}

\begin{abstract}
Resumo
A estratégia de modularização em produtos é mencionada como razão de diversos benefícios para as empresas. Este artigo tem como objetivo identificar na literatura os métodos para desenvolvimento da modularização em produtos com o intuito de avaliar sua aplicabilidade e ferramentas utilizadas, visando facilitar a escolha do método para cada empresa. Para atingir estes objetivos foi realizada uma revisão sistemática de acordo com a repetibilidade e importância indicada nos artigos. Como resultado foram identificados seis diferentes métodos para modularização de produtos. Como contribuição do artigo destacam-se a análise dos métodos com relação às etapas para formação dos módulos (decomposição, integração e avaliação) bem como a análise com relação a parâmetros de classificação quanto à área de atuação de cada método (variedade, geração e ciclo de vida). Por fim, este estudo oferece um guia para as empresas, contextualizando ambientes de aplicação e relacionando conhecimentos necessários para aplicação dos métodos.
\end{abstract}

Palavras-chave

Módulos. Design modular. Desenvolvimento de produtos. Design e desenvolvimento de produtos. Metodologia de design de produtos.

\section{Introdução}

Muitas empresas estão diante do problema de proporcionar maior variedade de produtos para o mercado, mas, ao mesmo tempo, mantendo a menor variedade possível entre os produtos de modo a manter economia de escala (Bi \& Zhang, 2001). Desta forma, a complexidade interna no desenvolvimento de novos produtos e serviços nas empresas tem crescido para satisfazer a variedade externa, necessária para sobreviver no mercado (Daniilidis et al., 2012). Esta situação traz à tona a necessidade de estratégias para lidar com o aumento da variedade para o mercado, que traz aumento de complexidade no desenvolvimento de produtos.

A estratégia de configuração de produtos denominada modularização foi primeiramente mencionada na literatura nos anos 60. Desde então, a modularização tem ganho destaque tanto na área acadêmica quanto na indústria. A abordagem de produto modular é amplamente aplicada no desenvolvimento e venda de computadores, que podem ser configurados conforme as necessidades específicas dos clientes a partir das escolhas de, por exemplo, diferentes capacidades de memória e de processadores.

Entre os benefícios alcançados pela modularização pode-se citar o desenvolvimento mais rápido de produtos, a redução do custo de desenvolvimento de produtos futuros (Stone et al., 2000a) e a utilização do mesmo módulo em múltiplos produtos permitindo variedade (Holtta-Otto \& De Weck, 2007). Além de outros aspectos como economia de escala, economia de custos com estoque e logística, flexibilidade na reutilização de componentes, redução do tempo de montagem e do tempo de produção e fabricação de módulos em paralelo (Jose \& Tollenaere, 2005). Bi \& Zhang (2001) também citam a dissociação de tarefas e a facilidade de aprimorar, manter, reparar e descartar o produto como benefícios da modularização. Além de todos estes benefícios, a modularização também 
facilita a introdução de novas tecnologias, permitindo atualização dos produtos (Gershenson et al., 2003). Algumas das desvantagens relacionadas ao projeto modular são alto investimento inicial, aumento da complexidade de desenvolvimento, falta de foco no cliente (como objetivar a redução de custo primordialmente e negligenciar as necessidades do cliente), riscos com relação à propriedade intelectual, assim como o desenvolvimento de produtos similares, devido a maior facilidade de cópia na reutilização de módulos (Kong et al., 2010).

Da mesma forma que o interesse pelo estudo do desenvolvimento de produtos modulares, a atividade de pesquisa e desenvolvimento de métodos e métricas para a modularização também tem crescido (Gershenson et al., 2004). 0 desenvolvimento de produtos modulares requer maior comprometimento da equipe envolvida, uma vez que necessita de mais experiência, coordenação, esforço, tempo, e é mais caro que o design de produtos tradicionais, pois considera a concepção de diversos produtos ao mesmo tempo (Jose \& Tollenaere, 2005). Conforme mencionado, a modularização tem o potencial de trazer grandes benefícios às empresas, porém, a divisão de produtos em módulos não é uma tarefa simples (Holmqvist \& Persson, 2003).

A forma como a divisão dos produtos em módulos é feita e a extensão em que o produto é modularizado influenciam muito os benefícios que a empresa poderá obter (Holmqvist \& Persson, 2003). Para isso, métodos e ferramentas são propostos para auxiliar o processo de modularização de produtos. Todos os métodos para a modularização têm o objetivo de agrupar componentes em módulos, porém, cada método foi desenvolvido a partir de um ponto de vista diferente e proveniente de diferentes áreas de aplicação (Daniilidis et al., 2011). No entanto, verifica-se baixa inserção e dificuldade de adaptação destes métodos e ferramentas aos processos das empresas. Esta característica é resultado da dificuldade em aplicar estas práticas na realidade do desenvolvimento de produto das empresas (Kahn et al., 2012). 0 objetivo deste trabalho é fazer um levantamento dos métodos de modularização por meio de revisão sistemática da literatura, com o intuito de entendê-los e classificá-los, visando facilitar o processo de tomada de decisão a respeito de que métodos e abordagens são mais adequados conforme a área de aplicação.

Depois da introdução, o artigo apresenta uma seção de terminologia e conceitos, na qual são explicitados conceitos pertinentes ao entendimento do trabalho, bem como são feitas relações entre estes conceitos. Em seguida, é apresentado o método de pesquisa para a revisão sistemática. Depois da aplicação dos passos constituintes da revisão sistemática, vinte e oito artigos foram encontrados apresentando métodos para a modularização de produtos e sendo posteriormente analisados conforme parâmetros de classificação encontrados na literatura. Em seguida, os métodos são apresentados de forma breve e decompostos em etapas. Na seção subsequente, de discussão sobre o tema, os métodos são abordados conforme as etapas para obtenção da modularização. Por fim, são tecidas as considerações finais a respeito do trabalho e apresentadas as lacunas de pesquisa.

\section{Terminologia e conceitos}

0 termo "módulo" é amplamente utilizado em contextos diferentes. Associados a este conceito foram encontrados na literatura outros termos-chave importantes para o entendimento da modularidade, como interface, arquitetura de produto, plataforma de produto, família de produto e customização em massa. Estes conceitos são fundamentais para o leitor no entendimento das publicações e linguagem relacionados à área de modularidade de produtos. $\mathrm{Na}$ área de design de produto não existe uma definição universalmente aceita para o conceito de módulo (Gershenson et al., 2004). Segundo Miller \& Elgard (1998), módulo é uma unidade funcional essencial e autocontida em relação ao produto do qual faz parte, possuindo interfaces padronizadas e interações que permitem, por meio de combinações, a composição de produtos (ex: produção de memória RAM de diferentes capacidades, cambiáveis de acordo com os objetivos do usuário). Para os mesmos autores, o termo modularidade é um atributo de um sistema relacionado com a sua estrutura e funcionalidade, e modularização é a atividade em que a estruturação em módulos ocorre (seleção e agrupamento de componentes em módulos). 0 termo autocontido, utilizado pelos autores, refere-se a uma função realizada dentro do módulo e limitada a ele.

Complementarmente, interfaces são as superfícies de contato entre os módulos e podem ser funcionais, mecânicas (conectores, plugs), e elétricas (sinais, força); interações descrevem relações de entrada e saída entre módulos e podem ser relações de energia, de informações, materiais ou espaciais (Miller \& Elgard, 1998). Segundo Stewart \& Yan (2008), independentemente da variedade de conceitos diferentes a respeito do tema, é possível dizer que as características principais relacionadas à modularidade são: independência estrutural; independência funcional; minimização de interfaces; e interações com outros módulos ou influências externas.

Nepal et al. (2005) defendem que a modularização tem por objetivo desenvolver arquiteturas de produto que contenham unidades fisicamente destacáveis, de 
forma a permitir que estas possam ser utilizadas em variações de um produto ou em linhas de produtos diferentes, o que ocorre por meio da padronização das interfaces. 0 termo arquitetura de produto, mencionado anteriormente, é o esquema pelo qual a função de um produto é atribuída aos componentes físicos (Ulrich, 1995). A arquitetura de um produto pode ser dividida em dois tipos: integral e modular. Arquitetura integral é definida como estrutura física em que os elementos funcionais são ligados a um único, ou a um pequeno número, de elementos físicos. Enquanto que arquiteturas modulares possuem subestruturas que têm correspondência de um-para-um com um subconjunto de um modelo funcional de produto (Stone et al., 2000a).

Outro conceito que permeia o estudo da modularidade é o conceito de plataforma de produto. Plataforma de produto é um conjunto comum de módulos compartilhados que é utilizado em todas as variantes de uma mesma familia (Zhang et al., 2006; Jose \& Tollenaere, 2005).

Por sua vez, uma família de produtos é um conjunto de produtos relacionados e derivados de uma plataforma comum para atingir alta variedade externa com o mínimo de variedade interna (Zhang et al., 2006). 0 desenvolvimento de família de produtos tem sido reconhecido como um meio efetivo para alcançar a economia de escala a fim de acomodar maior variedade de produtos em diferentes nichos de mercado (Du et al., 2001). Em uma família de produtos, os módulos que constituem a plataforma e, portanto, se repetem em todos os produtos, conferem a economia de escala, enquanto os demais módulos conferem aos produtos a diferenciação necessária para atender a diferentes usos ou a diferentes segmentos de mercado.

Um dos objetivos mais pertinentes com relação à adoção da modularidade e ao desenvolvimento de famílias de produtos é a customização em massa, isto é, a habilidade de prover produtos customizados por um valor similar aos produzidos em larga escala. Pine (1993) argumenta que a modularidade é chave para alcançar a customização em massa. Segundo Duray (2002), a modularidade é o aspecto-chave para alcançar o volume de escala (the 'mass'), enquanto o envolvimento do consumidor gera a customização. Da Silveira et al. (2001) defendem que produtos provenientes da customização em massa devem ser modulares, versáteis e constantemente renovados.

Sendo assim, a transição recente da produção em massa para a customização em massa tem forçado as empresas a desenvolverem famílias de produtos com uma plataforma comum para aumentar a variedade do design, diminuir o time-to-market e reduzir os custos dos produtos (Zhang et al., 2006).

Na Figura 1 são apresentados os conceitos discutidos nesta seção de forma gráfica, como um quebra-cabeças. 0 módulo é uma peça que, combinada a outras peças, forma o produto. Uma família de produtos é um conjunto de produtos formados por algumas peças que se repetem de um produto para o outro, sendo que a peça presente em todas as variantes é a plataforma de produto.

\section{Procedimentos metodológicos}

Para o levantamento qualitativo dos métodos existentes para a modularização no desenvolvimento do conceito do produto, foi utilizado o método de revisão sistemática da literatura proposto por Brereton et al. (2007). A revisão sistemática consiste em identificar, avaliar e interpretar as pesquisas relevantes relacionadas a um específico problema, tópico, área ou fenômeno de interesse. A seguir, são explicitadas as etapas componentes da revisão sistemática.

1. Questão de pesquisa: A questão formulada para guiar este estudo é: Quais são os métodos existentes para identificar, selecionar e avaliar conceitos em projetos modulares no desenvolvimento de produtos? Neste trabalho, adotou-se a definição de método de Holmqvist \& Persson (2003), como uma ferramenta de suporte para alcançar os objetivos estipulados da modularização.

2. Desenvolvimento de um protocolo de busca: 0 desenvolvimento de um protocolo de busca se inicia pela definição das palavras-chave. Assim como descrito por Brereton et al. (2007), foi
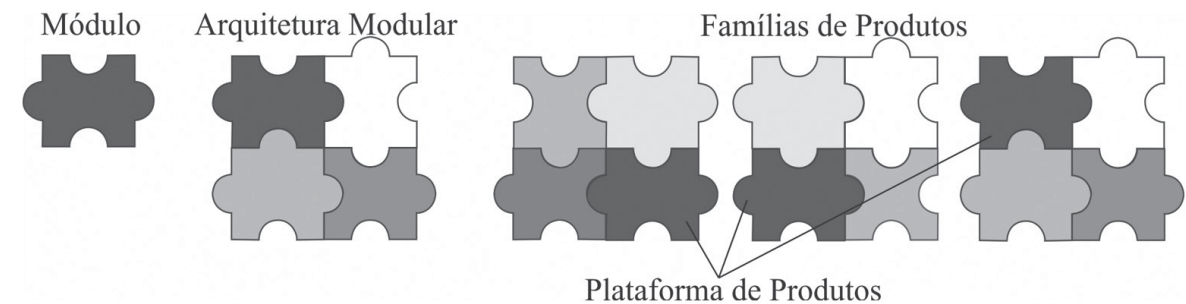

Figura 1. Associação de conceitos relacionados à literatura de modularidade. Fonte: Elaborado pelos autores. 
conduzida uma busca preliminar com o intuito de avaliar a quantidade de artigos relevantes, testar termos derivados da questão de pesquisa e revisar resultados de pesquisas já desenvolvidas. Nesta etapa, foi verificado que a busca pelo termo 'modularization methods' apresentou poucos estudos relevantes. Seguiu-se uma rodada de testes em que foi verificado que os melhores resultados advinham da busca por termos relacionados à área de desenvolvimento de produto, como o termo 'arquitetura de produto' e termos que ligavam a modularidade a seus objetivos, como a customização em massa, a geração de famílias e plataformas. Estes termos que permeiam o estudo da modularidade possibilitaram a criação de um string de busca mais amplo que permitiu acesso aos métodos e também a artigos que fazem uso desses métodos com vista a diferentes objetivos. 0 string utilizado foi (( "mass customization" OR "product platform" OR "product family" OR "product architecture") $\left.A N D \operatorname{modu} F^{*}\right)$. A busca foi realizada nas bases de dados Web of Science e Science Direct, em títulos, resumos e palavras-chave. Nas próprias bases de dados foram realizados refinamentos quanto ao idioma, selecionando o inglês, e no campo de áreas do conhecimento. A Tabela 1 resume os procedimentos desta revisão sistemática.

3. Inclusão de Literatura Cinzenta: Depois da leitura dos artigos, considerou-se a inclusão de dois artigos à revisão sistemática. 0 artigo de Pimmler \& Eppinger (1994) foi incluso por ser amplamente citado e utilizado entre os trabalhos encontrados na revisão, e sentiu-se a necessidade de recorrer ao artigo original para melhor descrever o método. Também foi incluso o método de Erixon (Ericsson
\& Erixon, 1999), referenciado por diversos trabalhos e publicado em forma de livro e tese de doutorado.

4. Critérios para a seleção de artigos: A avaliação dos artigos se deu por meio de um processo de triagem baseado em duas etapas, conforme descrito em Brereton et al. (2007). Na primeira etapa foram lidos títulos, resumos e palavras-chave e eliminados os artigos que claramente não tratavam da questão de pesquisa. Artigos cujo resumo e título não deixavam claro o escopo do trabalho foram mantidos para leitura na segunda etapa. Na segunda etapa os textos foram lidos por completo e foram considerados para o escopo desta pesquisa artigos que estavam de acordo com os critérios de inclusão e exclusão estabelecidos.

Foram inclusos os trabalhos que apresentam métodos para a modularização de projetos de produtos individuais. Foram excluídos os trabalhos que apresentam método para modularização de famílias e plataformas de produtos. A diferença entre estas abordagens é que os métodos para produtos individuais partem da análise de um produto inicial, criando módulos para este produto que podem ser utilizados em outros produtos de uma mesma família ou para atender a futuras demandas de mercado. Enquanto que os métodos para geração de famílias e plataformas consideram o desenvolvimento simultâneo de vários produtos de uma família e buscam por módulos que atuem como um denominador comum entre esses produtos.

Neste trabalho optou-se por analisar os métodos para modularização de forma mais restrita, considerando somente os métodos aplicáveis a arquiteturas de

Tabela 1. Resumo revisão sistemática.

Protocolo de Busca - Maio de 2014

\begin{tabular}{|c|c|c|}
\hline \multirow[t]{3}{*}{ ra identificar, selecionar e avaliar conceitos em projetos modulares $r$} & esenvolvimento & produtos? \\
\hline & \multicolumn{2}{|c|}{ Bases de dados } \\
\hline & Science Direct & Web of Science \\
\hline $\begin{array}{l}\text { String de busca: ((“mass customization” OR “product platform” OR “product family” OR “product } \\
\text { architecture”) AND modul”) em títulos, resumos e palavras-chave (sem restrição quanto à data de } \\
\text { publicação) }\end{array}$ & 88 & 720 \\
\hline Somente artigos de periódicos & 88 & 367 \\
\hline Lingua inglesa & 88 & 364 \\
\hline $\begin{array}{l}\text { Áreas de conhecimento: Operations Research Management Science, Eng. Manufacturing, } \\
\text { Eng. Industrial, Management, Computer Science Interdisciplinary Applications, Business, Eng. } \\
\text { Multidisciplinary e Planning Development }\end{array}$ & 88 & 297 \\
\hline Total & \multicolumn{2}{|c|}{385 artigos } \\
\hline Remoção dos artigos duplicados & \multicolumn{2}{|c|}{$(-44)=341$ artigos } \\
\hline Inclusão de literatura cinzenta: Ericsson \& Erixon (1999) e Pimmler \& Eppinger (1994) & \multicolumn{2}{|c|}{$(+2)=343$ artigos } \\
\hline $\begin{array}{l}\text { Seleção de Artigos, } 1^{\circ} \text { triagem: exclusão dos artigos que claramente não tratavam do escopo da } \\
\text { pesquisa }\end{array}$ & \multicolumn{2}{|c|}{$(343-240)=103$ artigos } \\
\hline $\begin{array}{l}\text { Seleção de Artigos, } 2^{\circ} \text { triagem: artigos que apresentam métodos para a modularização de arquiteturas } \\
\text { individuais }\end{array}$ & \multicolumn{2}{|c|}{28 artigos } \\
\hline Seleção de Artigos, $3^{\circ}$ triagem: exclusão dos métodos repetidos (consideração da fonte primária) & \multicolumn{2}{|c|}{6 métodos } \\
\hline $\begin{array}{l}\text { Extração de dados: parâmetros de classificação (Daniilidis et al., 2011); análise conforme as três fases } \\
\text { críticas (Holmqvist \& Persson, 2003) }\end{array}$ & \multicolumn{2}{|c|}{6 métodos } \\
\hline
\end{tabular}

Fonte: Elaborado pelos autores. 
produtos individuais, pois respondem diretamente à questão de pesquisa. Assim como no trabalho de Gershenson et al. (2004), os métodos para geração de famílias e plataformas, mesmo que possam ser utilizados para a modularização de produtos únicos, não foram considerados neste trabalho. Desta forma, o espaço de análise é delimitado, garantindo que as comparações sejam feitas entre métodos que trabalham o problema da modularização partindo de um mesmo ponto inicial: a consideração de uma única arquitetura de produto.

Com o objetivo de verificar a diversidade dos métodos foi realizada ainda uma terceira triagem, devido ao fato de que alguns artigos encontrados na revisão se utilizavam dos mesmos métodos para a modularização, resultando em seis diferentes métodos apresentados em 28 artigos.

5. Avaliação da qualidade dos estudos: Foram estabelecidos como critérios para o acesso da qualidade dos estudos a apresentação de etapa de decomposição da arquitetura e integração de componentes em módulos com suficiente detalhe, esclarecendo toda a sequência de atividades utilizada para obter a modularização.

Estes critérios visam avaliar os artigos com relação à integralidade da informação, excluindo os artigos que apresentavam resultados questionáveis ou incompletos. Nesta etapa foram excluídos os artigos que não apresentavam com detalhe o processo de modularização, como Huang \& Hsu (2011). Depois da avaliação de qualidade, restaram seis métodos que atenderam a todos os requisitos estabelecidos na revisão e que serão analisados neste artigo.

6. Extração de dados: A extração de dados tem o objetivo de coletar todas as informações necessárias para responder à questão e aos critérios de qualidade do estudo e precisa ser tabulada de maneira consistente com a questão de pesquisa, para realçar as similaridades e diferenças entre os estudos.

Os métodos foram analisados com relação ao framework proposto por Daniilidis et al. (2011), com relação a parâmetros de variedade, geração e ciclo de vida. Também foram analisados conforme a divisão proposta por Holmqvist \& Persson (2003), que considera três fases críticas para a modularização: a fase de decomposição, integração e avaliação. Estas três fases correspondem aos conceitos identificar, selecionar e avaliar utilizados na questão de pesquisa, e são melhor discutidos na continuidade deste artigo.

\section{Métodos para modularização}

Diferentes técnicas e metodologias já foram propostas na literatura para ajudar as empresas a desenvolver produtos modulares. Estudos mostram que a implementação de métodos formais de identificação e geração de módulos traz economia significativa de tempo e recursos se comparada à utilização de conhecimento e 'instinto natural' das equipes de desenvolvimento (Stewart \& Yan, 2008). Os seis métodos resultantes da revisão sistemática são apresentados na Tabela 2. A primeira coluna apresenta

Tabela 2. Métodos para modularização encontrados na literatura.

\begin{tabular}{|c|c|c|}
\hline Métodos & Objetivos & Artigos \\
\hline $\begin{array}{l}\text { Design Structure Matrix } \\
\text { - DSM }\end{array}$ & $\begin{array}{l}\text {... encontrar alternativas de arquitetura de forma a } \\
\text { otimizar a qualidade do design resultante e facilitar } \\
\text { as substanciais demandas de coordenação requeridas } \\
\text { quando os subsistemas interagem. }\end{array}$ & $\begin{array}{l}\text { Pimmler \& Eppinger (1994), Holtta \& Otto (2005), } \\
\text { Sered \& Reich (2006), Alizon et al. (2007), Holtta Otto } \\
\text { \& De Weck (2007), Yu et al. (2007), Meehan et al. } \\
\text { (2007), Park et al. (2008), Helmer et al. (2010), } \\
\text { Simpson et al. (2012), Cheng et al. (2012), Tilstra et al. } \\
\text { (2012), Bonjour et al. (2013), Li et al. (2013), Borjesson } \\
\text { \& Holtta-Otto (2014) }\end{array}$ \\
\hline $\begin{array}{l}\text { Modular Function } \\
\text { Deployment - MFD }\end{array}$ & $\begin{array}{l}\text {... alcançar uma modularização que não só atenda às } \\
\text { melhorias esperadas pela empresa e suporte a seleção } \\
\text { de diretrizes, mas também fortaleça a capacidade da } \\
\text { empresa de confrontar expansões futuras e demandas } \\
\text { divergentes. }\end{array}$ & $\begin{array}{l}\text { Ericsson \& Erixon (1999), Borjesson \& Holtta-Otto } \\
\text { (2014) }\end{array}$ \\
\hline Heuristic Model -HM & $\begin{array}{l}\text {... proporcionar uma abordagem sistemática para } \\
\text { identificar os módulos de um produto a partir de um } \\
\text { modelo funcional. }\end{array}$ & $\begin{array}{l}\text { Stone et al. (2000a, b), Dahmus et al. (2001), } \\
\text { Chandrasekaran et al. (2004), Stone et al. (2004), Zha } \\
\text { \& Sriram (2006), Zhuo et al. (2008) }\end{array}$ \\
\hline Design for Variety - DfV & $\begin{array}{l}\text {... desenvolver uma arquitetura dissociada que requer } \\
\text { menos esforços para o desenvolvimento de produtos } \\
\text { futuros. }\end{array}$ & Martin \& 1shii (2002), Simpson et al. (2012) \\
\hline $\begin{array}{l}\text { House of modular } \\
\text { Enhancement - HOME }\end{array}$ & $\begin{array}{l}\text {...desenvolver um método de design modular para } \\
\text { atender às questões relacionadas ao ciclo de vida ainda } \\
\text { na fase de design. }\end{array}$ & Sand et al. (2002) \\
\hline Fuzzy Logic Based - FLB & $\begin{array}{l}\text {...otimizar os atributos de performance dos módulos } \\
\text { potenciais enquanto modulariza a arquitetura do } \\
\text { produto ainda na fase de desenvolvimento de conceito. }\end{array}$ & Nepal et al. (2005) \\
\hline
\end{tabular}


os métodos e as abreviações pelas quais são referidos no restante do trabalho. Na última coluna, nomeada Artigos, são apresentados os artigos que se utilizam daquele método encontrados na revisão sistemática e, em negrito, o artigo primário (seminal) no qual o método foi proposto.

\subsection{Parâmetros de classificação}

Daniilidis et al. (2011) propõem um framework para classificação de métodos de modularização baseado em três parâmetros de diferenciação: variedade, geração e ciclo de vida. Com relação à variedade, os métodos são classificados em métodos para a arquitetura de produto individual, famílias de produto ou portfólios de produto. Com relação à geração, os métodos podem ser divididos em métodos para o desenvolvimento de novos produtos ou métodos para reengenharia (redesign) de produtos já existentes. Por fim, os métodos são classificados conforme aspectos do ciclo de vida por eles abordados, incluindo design, manufatura, uso e reciclagem. Estes três parâmetros são utilizados para caracterizar a área de aplicação de cada método e seus objetivos. Esta classificação é baseada na descrição dos métodos feita pelos próprios autores, bem como da análise da aplicação dos métodos encontrada na revisão sistemática (Figura 2).

Com relação ao primeiro parâmetro não houve diferenciação, pois foram escolhidos somente métodos que abordam produtos individuais para a análise. Com relação à fase de geração, todos os métodos analisados levam em conta a pré-existência de uma arquitetura a partir da qual será feito o redesign do produto com vista à modularização. Com relação a este parâmetro, nenhum trabalho foi encontrado para lidar com a modularização no desenvolvimento de novos produtos.
0 último parâmetro analisado foi o ciclo de vida, no qual os métodos foram classificados conforme a atenção a quatro diferentes aspectos: design, uso, manufatura e reciclagem. Dentre os quatro métodos, somente dois abordam todos estes aspectos: Modular Function Deployment (Ericsson \& Erixon, 1999) e House of Modular Enhancement (Sand et al., 2002). Estes métodos estão mais em sintonia com os objetivos da empresa, pois são estes objetivos que guiam o processo de modularização.

A classificação demonstrou similaridades entre os métodos avaliados, sendo que somente um dos parâmetros, a análise do ciclo de vida, apresentou diferenças entre os métodos. Desta forma, a análise dos métodos com relação às fases, assim como procedimentos adotados em cada fase, pode ser um diferencial na escolha do método por uma empresa. As particularidades de cada método, como decomposição funcional ou estrutural, uso de técnicas quantitativas ou qualitativas, entre outros, podem servir de guia para a escolha do método conforme as características do produto a ser modularizado.

\subsection{Decomposição dos métodos em etapas}

Os métodos para a modularização de produtos individuais possuem semelhanças e, por isso, podem ser analisados conforme etapas comuns para a obtenção do projeto modular. Holmqvist \& Persson (2003) apresentam três etapas, consideradas críticas, para a obtenção da modularidade: decomposição dos produtos em partes, integração dessas partes em módulos e avaliação do design resultante.

Na Tabela 3 os métodos (linhas) são analisados conforme as três fases críticas propostas por Holmqvist \& Persson (2003) e adotadas neste trabalho: decomposição, integração e avaliação (colunas).

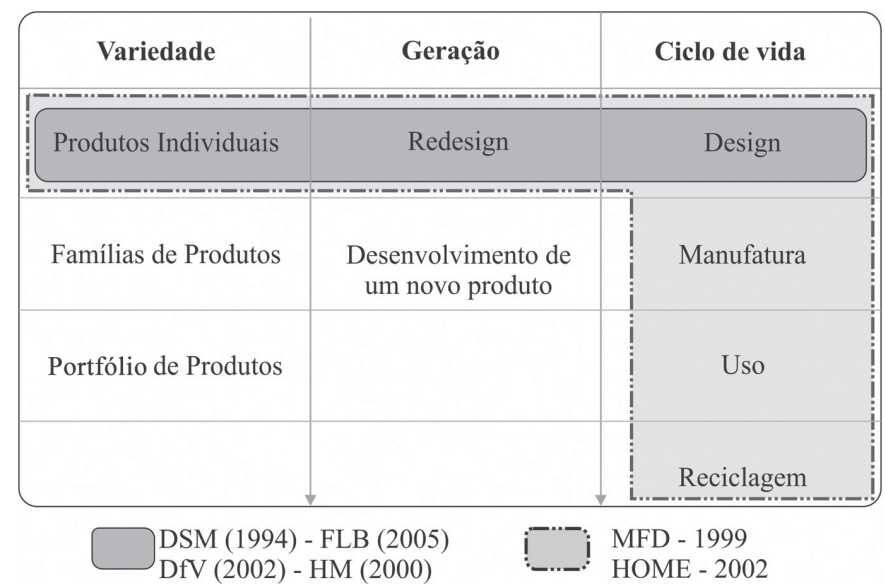

Figura 2. Parâmetros de classificação de Daniilidis et al. (2011). Fonte: Elaborado pelos autores. 
Tabela 3. Métodos para modularização segundo as 3 etapas críticas propostas por Holmqvist \& Persson (2003).

\begin{tabular}{|c|c|c|c|}
\hline & Decomposição & Integração & Avaliação \\
\hline \multirow{2}{*}{$\begin{array}{l}\text { DSM } \\
\text { Pimmler \& Eppinger (1994) }\end{array}$} & \multirow{2}{*}{$\begin{array}{l}\text { Decomposição do sistema em } \\
\text { elementos }\end{array}$} & $\begin{array}{l}\text { Documentação das interações entre } \\
\text { elementos }\end{array}$ & \\
\hline & & $\begin{array}{l}\text { Agrupamento dos elementos em } \\
\text { "chunks" de arquitetura e de equipe }\end{array}$ & \\
\hline \multirow{2}{*}{$\begin{array}{l}\text { MFD } \\
\text { Ericsson \& Erixon (1999) }\end{array}$} & Definição dos requisitos do cliente & \multirow{2}{*}{ - Geração de conceitos - matriz MIM } & Avaliação dos conceitos \\
\hline & Seleção das soluções técnicas & & Aprimoramento dos módulos \\
\hline $\begin{array}{l}\text { Método Heurístico } \\
\text { Stone et al. (2000a) }\end{array}$ & Decomposição funcional & $\begin{array}{l}\text { Aplicação de três heurísticas (fluxo } \\
\text { dominante, fluxo ramificado e fluxo } \\
\text { de conversão-transmissão) }\end{array}$ & \\
\hline \multirow[t]{3}{*}{$\begin{array}{l}\text { DfV } \\
\text { Martin \& 1shii (2002) }\end{array}$} & \multirow{3}{*}{$\begin{array}{l}\text { Geração do índice } \mathrm{GVl} \text { e } \mathrm{Cl} \text {. } \\
\text { Generational Variety lndex - } \\
\text { indicador da quantidade esperada de } \\
\text { redesenho necessário para que um } \\
\text { componente satisfaça as exigências } \\
\text { do mercado no futuro. } \\
\text { Coupling Index - indicador da força } \\
\text { de acoplamento: quanto mais forte } \\
\text { o acoplamento, maior a chance de } \\
\text { a mudança em um componente } \\
\text { requerer a mudança no outro. }\end{array}$} & Ordenar componentes & \\
\hline & & $\begin{array}{l}\text { Determinar onde modularizar }(\mathrm{Cl}) \text { e } \\
\text { onde padronizar }(\mathrm{GVI})\end{array}$ & \\
\hline & & $\begin{array}{l}\text { Desenvolver arquitetura } \\
\text { (componentes-funções/interfaces) }\end{array}$ & \\
\hline $\begin{array}{l}\text { HOME } \\
\text { Sand et al. (2002) }\end{array}$ & $\begin{array}{l}\text { Matriz de informação do design } \\
\text { modular - ciclo de vida, arquitetura e } \\
\text { requisitos funcionais }\end{array}$ & $\begin{array}{l}\text { Matriz EMIM - Algoritmo de } \\
\text { Agrupamento (MPA) }\end{array}$ & Análise do design modular \\
\hline \multirow[b]{2}{*}{$\begin{array}{l}\text { FLB } \\
\text { Nepal et al. (2005) }\end{array}$} & \multirow{2}{*}{$\begin{array}{l}\text { Processo de aquisição do } \\
\text { conhecimento (análise geral do } \\
\text { produto - identificação das variáveis } \\
\text { linguísticas...) }\end{array}$} & Inferência Fuzzy & \\
\hline & & $\begin{array}{l}\text { Modelo matemático baseado em } \\
\text { algoritmo de tecnologia de grupo }\end{array}$ & \\
\hline
\end{tabular}

Fonte: Elaborado pelos autores.

\section{Discussão}

Como já citado por Gershenson et al. (2004), todos os métodos para modularização requerem uma quantidade significativa de informação de entrada, cálculo e manipulações para chegar ao resultado esperado. Assume-se que a natureza dessas informações, bem como as manipulações e cálculos necessários, possam ser grandes influenciadores da escolha do método por uma determinada empresa.

A seção de discussão deste trabalho será feita baseada nas três fases críticas para a modularização: a decomposição, a integração e a avaliação, e também na implementação destes métodos. Todos os métodos passam pelo processo de decompor e integrar, porém, utilizando-se de ferramentas e estratégias diferentes para fazê-los. A etapa de avaliação, apesar de importante, não é encontrada em todos os métodos.

\subsection{Fase de decomposição}

Uma das abordagens mais encontradas entre os métodos é a decomposição funcional de produtos, também chamada de modelagem funcional. Decomposição funcional é o processo de quebrar a função global do produto em funções menores, em subfunções mais fáceis de resolver (Stone et al., 2000a). Estas subfunções fornecem uma descrição detalhada do que o produto deve fazer. Um dos aspectos mais importantes percebidos na análise dos métodos com relação à fase de decomposição é a existência de diferentes graus e tipos de decomposição. Os métodos analisados contemplam: decomposição funcional (Heurístico); decomposição funcional e/ou física (DSM, HOME e FLB); e a decomposição física em soluções técnicas (MFD) e em componentes (DfV).

A decomposição em soluções técnicas utilizada por Erixon (Ericsson \& Erixon, 1999) é semelhante à decomposição funcional de Stone et al. (2000a). 0 grau em que o produto é decomposto é o mesmo, relacionado às subfunções do produto. Contudo, a representação no método Heurístico utiliza as subfunções, enquanto no método MFD se utilizam as soluções técnicas que representam essas subfunções.

Quanto maior o grau de decomposição mais fácil encontrar as relações de um-para-um entre funções e partes físicas, mas também mais difícil é integrar essas partes, devido a maior quantidade de elementos a serem analisados (Holmqvist \& Persson, 2003). Pimmler \& Eppinger (1994) sugerem que a decomposição de produto seja feita em um nível de detalhe similar ao desejado para a arquitetura do produto.

\subsection{Fase de integração}

É nesta fase que o processo de modularização propriamente dito ocorre. Nesta fase os elementos identificados na fase de decomposição precisam ser 
agrupados para formar os módulos. Enquanto na fase de decomposição o que muda é o grau em que o produto é decomposto, na fase de integração os métodos se utilizam de estratégias diferentes para fazer a integração em módulos.

Uma das formas encontradas na literatura para trabalhar a fase de integração são as matrizes. No método DSM (Pimmler \& Eppinger, 1994), a matriz representa as relações entre os componentes/subsistemas, dispondo-os em linha e coluna e marcando interações que são utilizadas para definir os agrupamentos. Existem quatro principais tipos de interações: espacial, energia, informação e material. As interações são qualificadas em uma escala por meio de entrevistas e surveys com a equipe de desenvolvimento. Para formar os módulos um algoritmo de agrupamento é utilizado, reorganizando as linhas e colunas da matriz. Existem vários algoritmos que podem ser utilizados para agrupar os elementos da matriz DSM (Holmqvist \& Persson, 2003; Daniilidis et al., 2011).

No método MFD, proposto por Erixon (Ericsson \& Erixon, 1999), a modularização é realizada na matriz MIM (Module Identification Matrix), na qual cada solução técnica do produto é pontuada com relação às diretrizes de modularização, semelhante ao preenchimento do QFD (Quality Function Deployment), com pontuação 9 para forte relação, 3 para média relação, 1 para fraca relação e 0 quando a relação é inexistente. As diretrizes de modularização são motivos pelos quais as empresas desejam modularizar seus produtos (atualização, reciclagem, variedade, manutenção). Depois do preenchimento da matriz obtém-se o somatório das pontuações adquiridas pelas soluções e as soluções com maior pontuação são candidatas a tornarem-se módulos. As soluções com baixa pontuação não apresentam fortes motivos para tornarem-se módulos sozinhas e, portanto, podem ser integradas às demais soluções conforme indicações de similaridade apontadas na própria MIM. Uma outra abordagem para a formação de módulos a partir da matriz MIM é a utilização de algoritmos de agrupamento, assim como na matriz DSM.

No método HOME (Sand et al., 2002) a integração também é conduzida por matrizes. É gerada uma matriz, também chamada matriz MIM (Modular Information Matrix), que é resultado da combinação de três outras matrizes: Life Cycle Matrix, que relaciona componentes com outros componentes com relação ao ciclo de vida do produto; Product Architecture Matrix, que analisa as relações físicas entre os componentes do produto; e Function Structure Matrix, que analisa as relações entre a estrutura funcional do produto e seus componentes físicos. A Matriz MIM é gerada combinando as 3 matrizes acima citadas, e é transformada pelo Radial Axis Method em matriz
EMIM - Enhanced Modular Information Matrix. Um algoritmo de agrupamento é aplicado à matriz EMIM para a geração dos módulos.

No método Heurístico (Stone et al., 2000a) a modularização é feita a partir de três diferentes estratégias, chamadas heurísticas. Depois da decomposição funcional é criada uma representação do produto com seus fluxos de entrada e saída (fluxos de material, energia ou informação, que passam pelo produto formando um modelo funcional). A seguir, analisam-se cada fluxo e suas operações, criando cadeias de funções pelas quais este fluxo passa até a sua saída ou sua transformação em outro fluxo. As heurísticas deste método de análise consistem em identificar fluxos do produto e agrupar subfunções de acordo com as relações definidas por estes fluxos. As três heurísticas propostas são: (i) fluxo dominante, na qual se examina cada fluxo não ramificado de uma estrutura funcional e agrupa as subfunções percorridas até que ele saia do sistema ou se transforme em outro fluxo; (ii) fluxo ramificado, considera que um fluxo pode se ramificar formando cadeias de funções independentes, nas quais cada ramo de uma função paralela define um módulo potencial; (iii) fluxo de conversão-transmissão refere-se a um fluxo que pode ser convertido em outro, em que a subfunção de conversão-transmissão constitui um módulo.

No método DfV (Martin \& 1shii, 2002), são utilizados dois índices, o GVI (generational variety index, indicador da quantidade esperada de redesenho necessário para que um componente satisfaça as exigências do mercado no futuro) e o $\mathrm{Cl}$ (coupling index indica a força de acoplamento entre dois componentes, em que quanto mais forte for a relação de acoplamento, maior será a chance de a mudança em um componente requerer a mudança no outro) que fornecem para a equipe o entendimento dos fatores externos que provocam mudanças em um produto e como essas mudanças se propagam pela sua arquitetura. A modularização do produto tem relação direta com o índice $\mathrm{Cl}$ : analisando as relações entre os componentes é possível agrupá-los em um mesmo módulo, de forma que a sua mudança no futuro (para atender a novas necessidades, relacionado ao GVl) não afete todo o resto do produto. GVl e $\mathrm{Cl}$ são valores atribuídos pela equipe e baseados em fatores como as necessidades dos consumidores, confiabilidade de componentes e custos.

0 método FLB, de Nepal et al. (2005) utiliza-se de um processo de inferência fuzzy e de um modelo matemático. Os conceitos de lógica fuzzy podem ser utilizados para criar modelos matemáticos baseados em um conjunto de regras linguísticas. Depois da decomposição do produto são identificadas variáveis linguísticas de entrada (definidas pela equipe, como 
custo de interfaces, recursos de montagem...) e variáveis de saída (custo dos módulos, dado que o objetivo fixado é redução dos custos). Desenvolvem-se diretrizes de avaliação e regras do tipo SE-ENTÃO, para relacionar informações de entradas e saídas, formando um número de potenciais módulos, associadas ao custo destes módulos. Na sequência ocorre um processo de inferência fuzzy, e o processo de modularização do produto é obtido por meio de um modelo matemático baseado em um algoritmo de tecnologia de grupo, visando a minimização do custo de modularização de uma arquitetura de produto. Este método visa preencher uma lacuna de pesquisa apontada por Gershenson et al. (2004), que cita que os métodos para a modularização requerem informações que nem sempre estão disponíveis, principalmente nos estágios em que considerações a respeito do projeto modular são mais efetivas, isto é, nas fases iniciais do desenvolvimento.

\subsection{Fase de avaliação}

Tanto métodos para modularizar produtos quanto métodos mais amplos, que incluem a fase de modularização, envolvem a avaliação do design, que pode ser realizada com vista a diferentes perspectivas: função, flexibilidade, custos, complexidade (Bi \& Zhang, 2001). Isso porque a saída do método para modularização pode não ser um design factível. A avaliação precisa levar em conta todos os outros aspectos da realização do produto. Muitas vezes, o processo de integração utilizado no método leve a um design teoricamente adequado, mas que se apresente inviável quando confrontado a um processo de produção completo.

Entre os métodos estudados, o único método que apresenta uma etapa de avaliação bem estruturada é o MFD. 0 método MFD é composto de cinco etapas e as duas últimas etapas (análise de interfaces e aprimoramento intramódulos) são relacionadas à análise do design modular. A etapa número quatro diz respeito à análise de interfaces entre os módulos, que, além de clarificar as relações entre os módulos identificados na matriz MIM, serve de indicativo para o melhor sistema de montagem para o produto como um todo. A quinta etapa diz respeito a otimização intramódulo. Ericsson \& Erixon (1999) exemplificam a quinta etapa citando que se um módulo foi escolhido devido às suas fortes relações com manutenção e serviços, ele deve ser fabricado visando fácil desmontagem.

0 método HOME (Sand et al., 2002) também apresenta uma fase de avaliação do design modular, porém não é apresentado procedimento formal para que esta seja feita. Os módulos são analisados com relação aos componentes agrupados, de forma a verificar a viabilidade dos agrupamentos.

Os outros métodos não apresentam procedimentos formais para a avaliação do design resultante da fase de integração. Entretanto, todos os métodos sugerem que o projeto resultante seja avaliado e, se necessário, revisto.

\subsection{Implementação}

Todos os métodos analisados exigem da equipe de desenvolvimento amplo conhecimento do funcionamento e das características dos projetos dos produtos. Por consequência, a qualidade dos módulos finais depende da correta utilização das etapas propostas nos métodos e da experiência e conhecimento da equipe.

Alguns métodos apresentam uma implementação mais simples com relação ao passo a passo e às ferramentas utilizadas. 0 método DSM e Heurístico exigem da equipe de desenvolvimento a decomposição funcional e o mapeamento dos fluxos no produto, para posterior aplicação de um algoritmo de agrupamento (DSM) e das três estratégias de agrupamento (Heurístico). 0 método MFD também é de simples implementação, pois exige da equipe a decomposição do produto, o preenchimento da matriz MIM (similar ao QFD) e posterior soma ou aplicação de algoritmo de agrupamento para a geração dos módulos.

Os outros três métodos, DfV, HOME e FLB, apresentam uma implementação que emprega métodos/ ferramentas de forma integrada para a realização das etapas necessárias à modularização. 0 método HOME apresenta uma estrutura de matrizes que, além de exigir uma quantidade maior de matrizes (são geradas 11 matrizes no decorrer do método), se utiliza de um método para a transformação de matrizes (Radial Axis Method) e um algoritmo de agrupamento ao final. 0 método DFV exige uma série de etapas complexas para a sua implementação, pois necessita da utilização de diversos outros métodos e ferramentas para 0 cumprimento de suas etapas. A geração dos índices $\mathrm{GVl}$ e $\mathrm{Cl}$, utilizados para desenvolver a arquitetura do produto, contempla uma série de passos e ferramentas que incluem o QFD, a identificação e mapeamento de fluxos do produto e o Engineering Metric Target Values - EMTV. Vale ressaltar que o mapeamento de fluxos do produto é utilizado no DSM e método Heurístico diretamente para a geração dos módulos, enquanto no método DfV é utilizado em uma pequena parte de sua primeira etapa de geração de índices. De todos os métodos estudados, o método FLB é o que apresenta a maior quantidade de etapas, cálculos e manipulações para a geração dos módulos: exige 
a decomposição física e funcional, inferência fuzzy (que apresenta suas próprias regras e etapas), e, por final, um modelo matemático baseado em $P$-Mediam Group Technology Algorithm.

\section{Conclusões}

Este trabalho realizou o levantamento de métodos para modularização existentes na literatura com o objetivo de esclarecer os métodos e classificá-los, facilitando o entendimento e a escolha por parte das empresas do método mais adequado para seu caso específico. Para fazer o levantamento dos métodos para modularização existentes na literatura foi utilizado o método de revisão sistemática da literatura. Depois dos refinamentos necessários e da análise dos artigos, foram extraídos seis métodos para modularização de produtos individuais, posteriormente analisados.

Os métodos foram classificados conforme parâmetros descritos na literatura, bem como analisados com vista a diferentes etapas genéricas. A análise com relação às etapas genéricas e à classificação dos métodos segundo os parâmetros de variedade, geração e ciclo de vida tem por objetivo auxiliar as empresas na escolha de qual método é mais adequado para casos específicos. Nesta classificação pode-se perceber que os métodos estudados têm estrutura similar, pois referem-se a projetos de produto individuais e partem de uma arquitetura do produto definida anteriormente, sobre a qual será realizada a modularização. No projeto de novos produtos é necessária a etapa de definição da arquitetura do produto. 0 único parâmetro a apresentar diferenciação dentre os métodos selecionados neste artigo é relacionado ao ciclo de vida, no qual é avaliado o método que considera aspectos como uso e descarte do produto, além do design.

Para melhor discernimento dentre os métodos foi realizada uma análise das etapas constituintes, de forma a explicitar quais as competências e conhecimentos necessários para a aplicação de cada método. Nesta análise percebe-se que os métodos têm uma primeira fase bastante similar, na qual é decomposto o produto. A maior diferenciação entre os métodos está na estratégia utilizada para agrupar os componentes/partes dos produtos em módulos. Essa estratégia pode variar conforme o grau de decomposição feito na primeira etapa e pode ser baseada em diferentes critérios, como funções do produto, os objetivos da empresa e uma análise do ciclo de vida do produto. Neste trabalho considera-se que, com relação a etapas e ferramentas utilizadas, três métodos são de fácil e rápida implementação (DSM, MFD e Heurístico), e três métodos são mais complexos e exigem o suporte de outras ferramentas e métodos
(FLB, HOME e DfV), exigindo mais conhecimento da equipe e mais tempo para sua aplicação.

Como questões para pesquisas futuras, cita-se a necessidade de integração dos métodos para modularização a um modelo de processo de desenvolvimento de produtos, questão não abordada em nenhum dos trabalhos estudados. Assim como analisar, de forma mais aprofundada, quais as dificuldades encontradas pelas empresas na implementação dos métodos, e aprimoramentos que podem ser feitos para que eles se tornem mais facilmente aplicáveis, ao mesmo tempo que se mantém a qualidade do resultado. Outro ponto que necessita de maior atenção é o estudo dos métodos diante de projetos de produtos de diferentes complexidades, considerando a adaptação do método a projetos que apresentem diferentes características.

\section{Referências}

Alizon, F., Shooter, S. B., \& Simpson, T. W. (2007). Improving an existing product family based on commonality/diversity, modularity, and cost. Design Studies, 28(4), 387-409. http:// dx.doi.org/10.1016/j.destud.2007.01.002.

Bi, Z. M., \& Zhang, W. J. (2001). Modularity technology in manufacturing: taxonomy and issues. International Journal of Advanced Manufacturing Technology, 18(5), 381-390. http://dx.doi.org/10.1007/s001700170062.

Bonjour, E., Deniaud, S., \& Micaelli, J. (2013). A method for jointly drawing up the functional and design architectures of complex systems during the preliminary system-definition phase. Journal of Engineering Design, 24(4), 305-319. http://dx.doi.org/10.1080/09544828.2012.737457.

Borjesson, F., \& Holtta-Otto, K. (2014). A module generation algorithm for product architecture based on component interactions and strategic drivers. Research in Engineering Design, 25(1), 31-51. http://dx.doi.org/10.1007/s00163013-0164-2.

Brereton, P., Kitchenham, B. A., Budgen, D., Turner, M., \& Khalil, M. (2007). Lessons from applying the systematic literature review process within the software engineering domain. Journal of Systems and Software, 80(4), 571-583. http://dx.doi.org/10.1016/j.jss.2006.07.009.

Chandrasekaran, B., Stone, R. B., \& Mcadams, D. A. (2004). Developing design templates for product platform focused design. Journal of Engineering Design, 15(3), 209-228. http://dx.doi.org/10.1080/09544820410001647078.

Cheng, Q., Zhang, G., Gu, P., \& Shao, X. (2012). A product module identification approach based on axiomatic design and design structure matrix. Concurrent Engineering, Research and Applications, 20(3), 185-194. http://dx.doi. org/10.1177/1063293X12453350.

Da Silveira, G., Borenstein, D., \& Fogliatto, F. S. (2001). Mass customization: literature review and research directions. International Journal of Production Economics, 72(1), 1-13. http://dx.doi.org/10.1016/S0925-5273(00)00079-7.

Dahmus, J. B., Gonzales-Zugasti, J. P., \& Otto, K. N. (2001). Modular product architecture. Design Studies, 22(5), 409424. http://dx.doi.org/10.1016/S0142-694X(01)00004-7. 
Daniilidis, C., Enblin, V., Eben, K., \& Lindemann, U. (2011). A classification framework for product modularization methods. In Proceedings of the 18th International Conference on Engineering Design. Denmark.

Daniilidis, H., Bauer, W., \& Lindemann, U. (2012). Compendium for modular and platform based architecting. Procedia Computer Science, 8, 220-225. http://dx.doi.org/10.1016/j. procs.2012.01.046.

Du, X., Jiao, J., \& Tseng, M. M. (2001). Architecture of product family: fundamentals and methodology. Concurrent Engineering, Research and Applications, 9(4), 309-325. http://dx.doi.org/10.1177/1063293X0100900407.

Duray, R. (2002). Mass customization origins: mass or custom manufacturing? International Journal of Operations \& Production Management, 22(3), 314-328. http://dx.doi. org/10.1108/01443570210417614.

Ericsson, A., \& Erixon, G. (1999). Controlling design variants: modular product platforms. Dearborn: ASME Press.

Gershenson, J. K., Prasad, G. J., \& Zhang, Y. (2003). Product modularity: definitions and benefits. Journal of Engineering Design, 14(3), 295-313. http://dx.doi.org/10.1080/0954 482031000091068.

Gershenson, J. K., Prasad, G. J., \& Zhang, Y. (2004). Product modularity: measures and design methods. Journal of Engineering Design, 15(1), 33-51. http://dx.doi.org/10.1 080/0954482032000101731.

Helmer, R., Yassine, A., \& Meyer, C. (2010). Systematic module and interface definition using component design structure matrix. Journal of Engineering Design, 21(6), 647-675. http://dx.doi.org/10.1080/09544820802563226.

Holmqvist, T. K. P., \& Persson, M. L. (2003). Analysis and Improvement of Product Modularization Methods: Their Ability to Deal with Complex Products. Systems Engineering, 6(3), 195-209. http://dx.doi.org/10.1002/sys.10046.

Holtta, K. M. M., \& Otto, K. N. (2005). Incorporating design effort complexity measures in product architectural design and assessment. Design Studies, 26(5), 463-485. http:// dx.doi.org/10.1016/j.destud.2004.10.001.

Holtta-Otto, K., \& De Weck, O. (2007). Degree of modularity in engineering systems and products with technical and business constraints. Concurrent Engineering, Research and Applications, 15(2), 113-126. http://dx.doi. org/10.1177/1063293X07078931.

Huang, M. S., \& Hsu, M. K. (2011). Modular design applied to beverage-container injection molds. International Journal of Advanced Manufacturing Technology, 53(1-4), 1-10. http://dx.doi.org/10.1007/s00170-010-2796-y.

Jose, A., \& Tollenaere, M. (2005). Modular and platform methods for product family design: literature analysis. Journal of Intelligent Manufacturing, 16(3), 371-390. http://dx.doi. org/10.1007/s10845-005-7030-7.

Kahn, K. B., Barczak, G., Nicholas, J., Ledwith, A., \& Perks, H. (2012). An examination of new product development best practice. Journal of Product Innovation Management, 29(2), 180-192. http://dx.doi.org/10.1111/j.1540-5885.2011.00888.x.

Kong, F. B., Ming, X. G., Wang, L., Wang, X. H., \& Wang, P. P. (2010). On modular products development. Concurrent Engineering, Research and Applications, 1744), 291-300. http://dx.doi.org/10.1177/1063293X09353974.

Li, Z., Cheng, Z., Feng, Y., \& Yang, J. (2013). An integrated method for flexible platform modular architecture design. Journal of Engineering Design, 24(1), 25-44. http://dx.doi. org/10.1080/09544828.2012.668614.
Martin, M. V., \& Ishii, K. (2002). Design for variety: developing standardized and modularized product platform architectures. Research in Engineering Design, 13, 213-235. http://dx.doi. org/10.1007/s00163-002-0020-2.

Meehan, J. S., Duffy, A. H. B., \& Whitfield, R. 1. (2007). Supporting 'Design for Re-use' with modular design. Concurrent Engineering, Research and Applications, 15(2), 141-155. http://dx.doi.org/10.1177/1063293X07079319.

Miller, T. D., \& Elgard, P. (1998). Defining modules, modularity and modularization: evolution of the concept in a historical perspective. In Proceeding of the 13th IPS Research Seminar, Denmark.

Nepal, B., Monplaisir, L., \& Singh, N. (2005). Integrated fuzzy logic-based model for product modularization during concept development phase. International Journal of Production Economics, 96(2), 157-174. http://dx.doi. org/10.1016/j.jpe.2004.03.010.

Park, J., Shin, D., Insun, P., \& Hyemi, H. (2008). A product platform concept development method. Journal of Engineering Design, 19(6), 515-532. http://dx.doi. org/10.1080/09544820802043583.

Pimmler, T. U., \& Eppinger, S. D. (1994). Integration analysis of product decompositions. In Proceedings of the ASME Design Theory and Methodology Conference, Minneapolis, USA.

Pine, B. J. (1993). Mass customization: the new frontier in business competition. Boston: Harvard Business School Press.

Sand, J. C., Gu, P., \& Watson, G. (2002). HOME: House Of Modular Enhancement- a tool for modular product redesign. Concurrent Engineering, Research and Applications, 10(2), 153-164. http://dx.doi.org/10.1177/1063293X02010002638.

Sered, Y., \& Reich, Y. (2006). Standardization and modularization driven by minimizing overall process effort. Computer Aided Design, 38(5), 405-416. http://dx.doi.org/10.1016/j. cad.2005.11.005.

Simpson, T. W., Bobuk, A., Slingerland, L. A., Brennan, S., Logan, D., \& Reichard, K. (2012). From user requirements to commonality specifications: an integrated approach to product family design. Research in Engineering Design, 23(2), 14-153. http://dx.doi.org/10.1007/s00163-011-0119-4.

Stewart, B., \& Yan, X. (2008). Modular product family development within a SME. Global Design to Gain a Competitive Edge, 21-30. http://dx.doi.org/10.1007/978-1-84800-239-5_3.

Stone, R. B., McAdams, D. A., \& Kayyalethekkel, V. J. (2004). A product architecture-based conceptual DFA technique. Design Studies, 25(3), 301-325. http://dx.doi.org/10.1016/j. destud.2003.09.001.

Stone, R. B., Wood, K. L., \& Crawford, R. H. (2000a). A heuristic method for identifying modules for product architectures. Design Studies, 21(1), 5-31. http://dx.doi.org/10.1016/ S0142-694X(99)00003-4

Stone, R. B., Wood, K. L., \& Crawford, R. H. (2000b). Using quantitative functional models to develop product architectures. Design Studies, 21(3), 239-260. http:// dx.doi.org/10.1016/S0142-694X(99)00008-3.

Tilstra, A. H., Seepersad, C. C., \& Wood, K. L. (2012). A High Definition design structure matrix (HDDSM) for the quantitative assessment of product architecture. Journal of Engineering Design, 23, 767-789. http://dx.doi.org/1 $0.1080 / 09544828.2012 .706748$. 
Ulrich, K. (1995). The role of product architecture in the manufacturing firm. Research Policy, 24(3), 419-449. http:// dx.doi.org/10.1016/0048-7333(94)00775-3.

Yu, T. L., Yassine, A. A., \& Goldberg, D. E. (2007). An information theoretic method for developing modular architectures using genetic algorithms. Research in Engineering Design, 18(2), 91-109. http://dx.doi.org/10.1007/s00163-007-0030-1.

Zha, X. F., \& Sriram, R. D. (2006). Platform-based product design and development: a knowledge-intensive support approach. Knowledge-Based Systems, 19(7), 524-543. http://dx.doi.org/10.1016/j.knosys.2006.04.004.
Zhang, W. Y., Tor, S. Y., \& Britton, G. A. (2006). Managing modularity in product family design with functional modeling. International Journal of Advanced Manufacturing Technology, 30(7-8), 579-588. http://dx.doi.org/10.1007/ s00170-005-0112-z.

Zhuo, L., San, W. Y., \& Seng, L. K. (2008). Integrated approach to modularize the conceptual product family architecture. International Journal of Advanced Manufacturing Technology, 36(1-2), 83-96. http://dx.doi.org/10.1007/ s00170-006-0805-y.

\title{
Selection of modularization methods in product development: systematic review
}

\begin{abstract}
The strategy of product modularization is seen as a means to provide several benefits for companies. The objective of this paper is to identify in the literature the methods for developing modularization in products and to assess the applicability of and tools used by these methods, aiming at favoring the method that is the most suitable for each company. To achieve these objectives, a systematic review was performed according to the repeatability and importance described in the papers. Six different methods were identified for product modularization. This paper presents an analysis of the methods in relation to the module formation steps (decomposition, integration and evaluation) and provides an analysis related to classification parameters regarding the field of each method (variety, generation and life cycle). Finally, this study offers guidelines for companies, places application environments into context, and relates the knowledge necessary for applying those methods.
\end{abstract}

\section{Keywords}

Module. Conceptual design. Product development. Product design and development. Product design methodology. 